\title{
A ilusão da máscara côncava percebida por idosos
}

\author{
The hollow-face illusion perceived by the elderly
}

La ilusión de la máscara cóncava percibida por ancianos

Mateus Lopes de Carvalho ${ }^{1 *}$, Maria Amélia Cesari Quaglia1, Marcos Santos de Oliveira1.

\section{RESUMO}

Objetivo: Investigar diferenças na percepção visual de idosos, comparativamente a pessoas de faixa etária menor em relação à ilusão da máscara côncava. Métodos: Utilizou-se o delineamento pré-experimental de comparação com grupo estático. As amostras foram selecionadas acidentalmente. Participaram 42 idosos e 73 pessoas de faixa etária menor. A máscara côncava foi apresentada binocularmente aos participantes a uma distância de três metros e em duas condições de iluminação: por cima e por baixo. A análise dos dados foi feita com um software estatístico e o nível de significância considerado foi $5 \%$. O estudo foi aprovado por Comissão de Ética. Resultados: Não houve diferenças significativas entre os grupos no primeiro indicador da percepção visual de profundidade (côncavo ou convexo), independentemente da condição de iluminação (por cima, $p=0,26$; por baixo, $p=0,37$ ). No segundo indicador (julgamento categórico), não houve diferenças significativas entre os grupos (por cima, $p=0,97$; por baixo, $p=0,26$ ). No terceiro indicador (estimativa métrica), os resultados foram semelhantes (por cima, $p=0,48$; por baixo, $p=0,67$ ). Conclusão: As pessoas de faixa etária menor, tanto quanto os idosos, foram suscetíveis à ilusão da máscara côncava.

Palavras-chave: Percepção de profundidade, Idosos, Percepção visual.

\begin{abstract}
Objective: Investigate differences in visual perception of elderly people, comparatively to people of lower age group in relation to hollow-face illusion. Methods: A pre-experimental design of comparison with a static group was used. The samples were selected accidentally. 42 elderly people and 73 people of younger age participated. The concave mask was binocularly presented to the participants at a distance of three meters and in two lighting conditions: above and below. Data analysis was carried using a statistical software and the considered level of significance was $5 \%$. The study was approved by the Ethics Committee. Results: There were no significant differences between the groups in the first indicator of visual depth perception (concave or convex), regardless of lighting condition (above, $p=0.26$; below, $p=0.37$ ). In the second indicator (categorical judgment), there were no significant differences between the groups (above, $p=0.97$; below, $p=0.26$ ). In the third indicator (metric estimate), the results were similar (above, $p=0.48$; below, $p=0.67$ ). Conclusion: People in the younger age group, as well as the elderly, were susceptible to hollow-face illusion.
\end{abstract}

Keywords: Depth Perception, Elderly, Visual perception.

\section{RESUMEN}

Objetivo: Investigar diferencias en la percepción visual de ancianos, comparativamente a personas de franja etaria más baja en relación a la ilusión de la máscara cóncava. Métodos: Se utilizó la delineación preexperimental de comparación con grupo estático. Las muestras fueron seleccionadas accidentalmente. Participaron 42 ancianos y 73 personas de franja etaria más baja. La máscara cóncava fue presentada binocularmente a los participantes a una distancia de tres metros en dos condiciones de iluminación: por encima y por debajo. El análisis de datos fue hecho con software estadístico; el nivel de significancia considerado fue del 5\%. El estudio fue aprobado por Comisión de Ética. Resultados: No hubo diferencias significativas entre los grupos del primer indicador de la percepción visual de profundidad (cóncavo o convexo), independientemente de la condición de iluminación (por encima, $p=0,26$; por debajo, $p=0,37$ ). El segundo indicador (juzgamiento categórico) no presentó diferencias significativas entre los grupos (por

${ }^{1}$ Universidade Federal de São João del-Rei. São João del-Rei - MG.

*E-mail: mateuscarvalho.psicologia@gmail.com

SUBMETIDO EM: 6/2020

ACEITO EM: 6/2020

PUBLICADO EM: 8/2020

REAS/EJCH | Vol.12(11) | e4129 | DOI: https://doi.org/10.25248/reas.4129.2020 Página 1 de 9 
encima, $p=0,97$; por debajo, $p=0,26$ ). El tercer indicador (estimativa métrica) presentó resultados semejantes (por encima, $p=0,48$; por debajo, $p=0,67$ ). Conclusión: Las personas de franja etaria más baja, tanto como los ancianos, fueron susceptibles a la ilusión de la máscara cóncava.

Palabras clave: Percepción de Profundidad, Ancianos, Percepción visual.

\section{INTRODUÇÃO}

A velhice ou terceira idade, nos Estados Unidos, é compreendida como uma etapa do desenvolvimento humano que, cronologicamente, começa aos 65 anos (PAPALIA EE e FELDMAN RD, 2012). No Brasil, entretanto, conforme o Estatuto do Idoso, em seu Artigo 1, idoso é toda pessoa com idade igual ou superior a 60 anos (MINISTÉRIO DA SAÚDE, 2013).

O envelhecimento populacional aumentou nos últimos anos e a tendência é que se amplie ainda mais. Estima-se que, nas próximas décadas, o Brasil se situe entre os seis países com o maior número de pessoas acima de 60 anos (MACEDO BG, et al., 2008). Esse crescimento da população idosa aponta para a necessidade de reformulação no sistema social como um todo, principalmente no âmbito da saúde e da previdência social. Isso porque os idosos compõem o grupo de pessoas que consomem mais medicamentos, devido às doenças crônicas que são mais presentes nessa etapa de vida (BALDONI AO e PEREIRA LRL, 2011).

Pode ser que a velhice seja um momento para a realização de projetos pretendidos que não se concretizaram em etapas anteriores (DEBERT GG, 1999; PAPALIA EE e FELDMAN RD, 2012). Por outro lado, é inevitável o desgaste do corpo de maneira geral e, com isso, problemas e limitações diversos podem ocorrer (SANTOS FG, et al., 2009). Dentre as limitações na velhice, o sistema visual é um dos primeiros a se deteriorar, gerando comprometimentos na percepção visual, como alterações na percepção de profundidade e cores, sensibilidade ao contraste e adaptação ao escuro, além de doenças oftalmológicas, como catarata e glaucoma (MACEDO BG, et al., 2008).

A Função de Sensibilidade ao Contraste (FSC) tem sido muito pesquisada na população idosa, devido alterações nesta capacidade em consequência do avanço da idade. Em uma pesquisa envolvendo 16 voluntários, Costa TL, et al. (2009) encontraram diferenças significativas entre adultos jovens em relação à FSC. Os idosos necessitaram de mais brilho para perceber o contraste, quando comparados com pessoas mais jovens.

O reconhecimento de face, sendo a capacidade de visualizar rostos, memorizar e, posteriormente, recordar, é outro aspecto que tem sido estudado, observando-se diferenças nessa capacidade em relação à idade. Conforme Lamont AC, et al. (2005), com o declínio da idade, a capacidade de memorização e, consequentemente, o reconhecimento de faces diminui. Por isso, os idosos apresentam menos precisão no reconhecimento de faces, se comparados às pessoas mais jovens.

Apesar de haver muitas pesquisas envolvendo a percepção visual de idosos em comparação as pessoas mais jovens, não foi encontrado na literatura pesquisas comparando idosos e jovens quanto ao fenômeno de ilusão da máscara côncava. Nesta ilusão, ao apresentar um reverso de uma máscara facial para um indivíduo, a face é vista como convexa, como uma face normal, protusa. Isso ocorre devido à predominância dos processos top-down sobre os processos bottom-up (GREGORY RL, 1997a; GREGORY RL e GOMBRICH $\mathrm{EH}, 1980$ ). Essa ilusão ocorre com mais força, possivelmente, por causa da familiaridade da máscara com a face humana, cujo reconhecimento, ao longo da evolução, teve valor de sobrevivência para o homem (DARWIN C, 1871/1998).

Alguns achados na área de investigação da ilusão da máscara côncava apontaram que algumas condições ou situações interferem na percepção do fenômeno. A esquizofrenia, por exemplo, é um desses fatores (SCHNEIDER U, et al., 2002; DIMA D, et al., 2009; DIMA D, et al., 2011).

A síndrome de abstinência alcoólica é outro fator que desequilibra os processos top-down, necessários para a inversão da profundidade, assim como a privação de sono (SCHNEIDER U, et al., 1996a; SCHNEIDER U, et al., 1996b). Por outro lado, alguns estudos possibilitaram a comparação de indivíduos com faixas etárias 
distintas, como crianças e adultos. Os resultados da pesquisa de Corrow S, et al. (2011), por exemplo, evidenciou que até mesmo crianças de seis meses percebem a ilusão da máscara côncava em condição monocular.

Embora exista um considerável número de estudos sobre a ilusão da máscara côncava com diferentes populações, ainda são escassos os estudos que visam comparar grupos de diferentes faixas etárias. Particularmente, não foram encontradas pesquisas que tivessem como objetivo comparar adultos mais jovens e idosos em relação ao fenômeno em questão, apesar da possível deterioração do sistema visual em decorrência da idade. Com isso, esta pesquisa teve como objetivo investigar a percepção binocular do fenômeno de ilusão da máscara côncava em pessoas idosas, comparativamente a adultos de menor faixa etária.

\section{MÉTODOS}

\section{Delineamento}

Foi utilizado o Delineamento Pré-experimental de Comparação com Grupo Estático, em que os grupos comparados são naturais, não havendo introdução de uma variável experimental. Esse tipo de delineamento é considerado como uma pesquisa correlacional, porque permite mostrar a relação existente entre as variáveis estudadas, mas não a relação causal entre elas. Essas variáveis são do tipo organísmicas ou dos sujeitos (SELLTIZ C, et al., 2007).

\section{Participantes}

Participaram deste estudo duas populações-alvo: idosos e pessoas de faixa etária menor. Todos os participantes apresentaram acuidade visual normal (6/6) aferida por meio da Escala Optométrica Snellen e assinaram o Termo de Consentimento Livre e Esclarecido. Foram excluídos, de ambas as populações, os indivíduos: 1) que relataram uso de substâncias psicotrópicas ou álcool; 2) com diagnósticos de transtorno depressivo maior, esquizofrenia ou demências; e 3) aqueles que não compreenderam as questões e instruções dos instrumentos de medida.

\section{Material e equipamento}

Foi construída uma caixa de madeira, com: $27 \mathrm{~cm}$ de largura; $42 \mathrm{~cm}$ de altura e $12 \mathrm{~cm}$ de profundidade. Ela foi pintada de preto, por dentro e por fora, com o objetivo de impedir a refletância de luz. Na parte interna e central da caixa, foi colocada uma máscara côncava na posição vertical, de tamanho reduzido, da face obtida de uma cabeça de boneca comercial de plástico, com as seguintes medidas aproximadamente: $8 \mathrm{~cm}$ de altura; $6 \mathrm{~cm}$ de largura; e $3 \mathrm{~cm}$ de profundidade. A máscara foi pintada de bege, com olhos, boca e sobrancelhas demarcadas. Na parte frontal da caixa, foi feita uma abertura centralizada com $13 \mathrm{~cm}$ de largura e $18 \mathrm{~cm}$ de altura para possibilitar a visualização da máscara. Foram colocadas, ainda, duas lâmpadas de 15 volts no interior da caixa, uma na parte superior e outra na parte inferior, para iluminar a máscara.

\section{Procedimentos}

Os participantes do estudo foram informados acerca dos objetivos e aspectos éticos da pesquisa. O Termo de Consentimento Livre e Esclarecido foi apresentado, lido e assinado. Os participantes tiveram sua acuidade visual avaliada e responderam um questionário clínico. Após esta etapa, os participantes foram instruídos a observar a máscara côncava no interior do equipamento, binocularmente, em uma distância de três metros. Em seguida, foram avaliados três indicadores da percepção de profundidade ou relevo da máscara côncava. No primeiro indicador, questionou-se os participantes se a máscara foi vista por eles como côncava ou convexa. Para facilitar a compreensão dos participantes, os termos côncava e convexa foram substituídos, respectivamente, por voltada "para dentro" e "para fora".

No segundo indicador, os participantes foram solicitados a qualificar o nível de profundidade ou relevo percebido em cinco opções de respostas: muito côncava, côncava, plana, convexa e muito convexa. Para finalizar, no terceiro indicador, pediram-se aos participantes que quantificassem o nível de profundidade ou 
relevo percebido. Uma trena retrátil, do lado contrário, sem a numeração, foi apresentada aos participantes e eles foram solicitados a estimar a distância da ponta do nariz à base da máscara. Essa estimativa foi transformada em centímetros. Os três indicadores foram avaliados com a máscara sendo iluminada por cima e iluminada por baixo, aleatoriamente.

\section{Técnicas estatísticas}

Os dados foram analisados utilizando-se o Software Statistical Package for Social Sciences (SPSS), versão 22.0. O nível de significância considerado para as análises estatísticas foi $5 \%$. Para calcular as porcentagens e médias, utilizou-se a estatística descritiva. No primeiro indicador, foi utilizado o Teste Exato de Fisher para verificar se houve diferenças entre os grupos e o Teste Qui-quadrado de Pearson para analisar os grupos internamente em função do modo de iluminação da máscara. No segundo indicador, para comparar os grupos e analisá-los internamente, segundo o modo de iluminação da máscara, foi utilizado o Teste Quiquadrado de Pearson. Por fim, no terceiro indicador, utilizou-se o teste Kolmogorov-Smirnov para verificação da normalidade. Além disso, foram usados o teste $U$ de Mann-Whitney para comparação dos grupos e o teste Wilcoxon para analisar os grupos internamente conforme a direção da fonte de iluminação.

\section{Considerações éticas}

O presente estudo recebeu aprovação da Comissão de Ética em Pesquisa Envolvendo Seres Humanos da Universidade Federal de São João del-Rei, conforme CAAE: 76827417.7.000.5151. Foram seguidos todos os procedimentos éticos recomendados para a realização de pesquisas com seres humanos de acordo com as diretrizes da Resolução n. 466/2012 do Conselho Nacional de Saúde.

\section{RESULTADOS}

Esta pesquisa contou com duas amostras selecionadas acidentalmente. Uma delas foi composta por 42 idosos, sendo 8 pessoas do sexo masculino e 34 do sexo feminino, com média de idade de 67 anos $(D P=$ 4,2). A amostra da população de pessoas de faixa etária menor foi constituída de 73 indivíduos, sendo 28 do sexo masculino e 45 do sexo feminino, com média de idade de 23 anos $(D P=3,9)$.

No primeiro indicador da percepção de profundidade ou relevo da máscara côncava, com a iluminação na parte superior da máscara, $54,8 \%$ dos observadores do grupo de idosos perceberam a máscara como côncava e 45,2\% perceberam a máscara como convexa. Em relação aos observadores do grupo de pessoas com faixa etária menor, $46,6 \%$ perceberam a máscara como côncava e $53,4 \%$ perceberam a máscara como convexa.

No modo de iluminação por baixo da máscara, $42,9 \%$ dos idosos perceberam a máscara como côncava e $57,1 \%$ perceberam a máscara como convexa. No que se refere ao grupo de pessoas de faixa etária menor, $47,9 \%$ dos observadores perceberam a máscara como côncava e $52,1 \%$ perceberam a máscara como convexa (Figura 1).

Foram realizadas, também, análises estatísticas para verificar se houve diferenças relevantes entre os grupos nos dois modos de iluminação. No modo de iluminação por cima, não foram encontradas diferenças significativas entre os grupos $(p=0,26)$. No modo de iluminação por baixo, os resultados foram semelhantes, não houve diferença estatisticamente relevante entre os grupos $(p=0,37)$.

Os grupos também foram analisados internamente a fim de descobrir se houve diferenças significativas nos casos de inversão ou não da profundidade da máscara côncava, em função dos dois modos de iluminação (por cima e por baixo). No grupo de idosos, não foram reveladas diferenças significativas nos casos de inversão em função do modo de iluminação $(p=0,28)$. Semelhantemente, não foram encontradas diferenças significativas no grupo de pessoas de faixa etária menor $(p=0,87)$. 
Figura 1 - Porcentagens da percepção de profundidade (côncava) ou relevo (convexa) da máscara côncava.

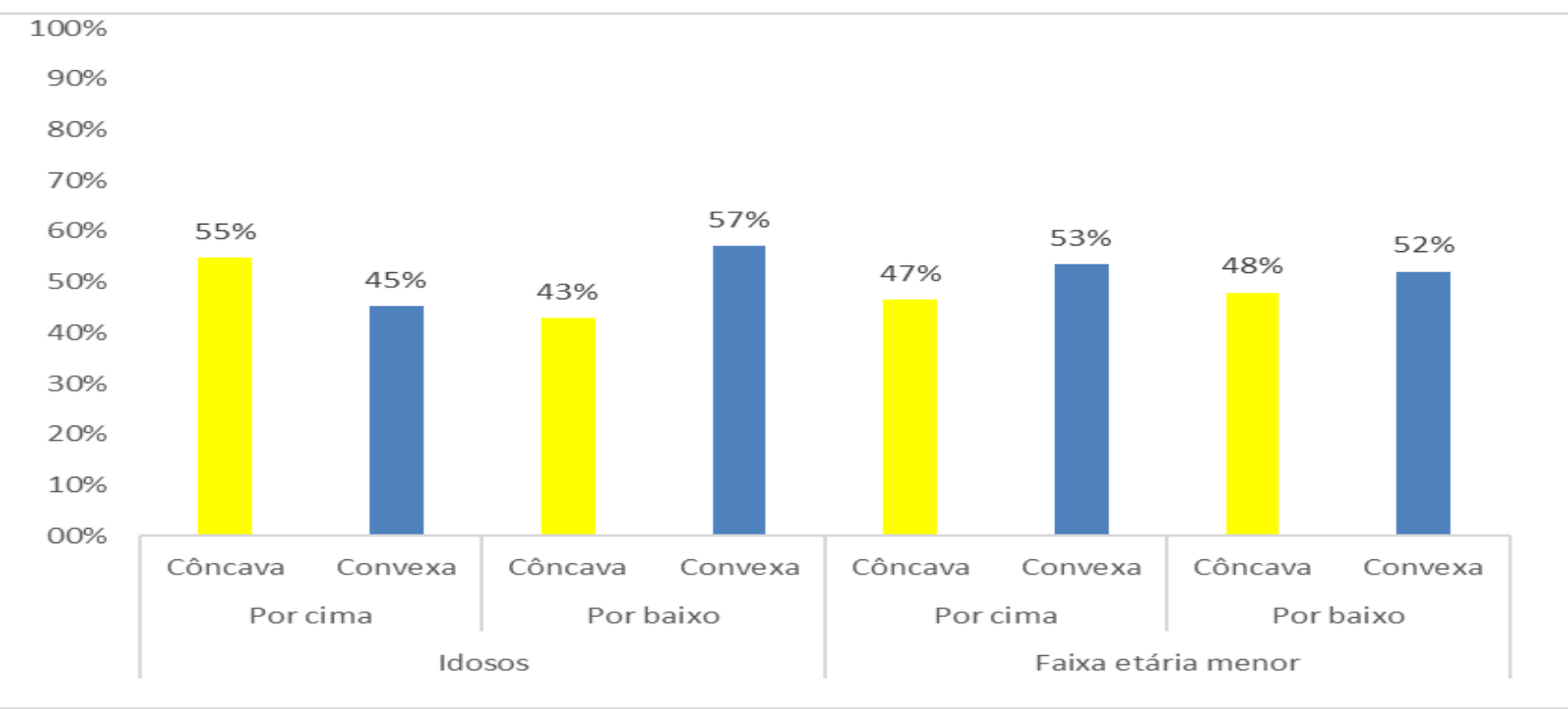

Fonte: Carvalho ML, et al., 2020.

$\mathrm{Na}$ análise do segundo indicador, ao utilizar o Teste Qui-Quadrado de Pearson para avaliar os julgamentos categóricos, tanto no modo de iluminação por cima, quanto por baixo, quatro células apresentaram uma frequência esperada menor que cinco. Por esta razão, os dados foram reagrupados, nos dois modos de iluminação. Assim, as categorias muito côncava e côncava se tornaram a categoria côncava. A categoria plana não sofreu alteração. E as categorias convexa e muito convexa foram agrupadas na categoria convexa.

Assim, serão apresentados apenas os dados referentes a esse novo agrupamento. No modo de iluminação por cima, dos observadores do grupo de idosos, os resultados dos julgamentos categóricos foram os seguintes: $50 \%$ na categoria côncava; $7,1 \%$ na categoria plana; e 42,9\% na categoria convexa. Em relação ao grupo de pessoas de faixa etária menor, os dados foram os seguintes: $50,7 \%$ de julgamentos na categoria côncava; $8,2 \%$ na categoria plana; e $41,1 \%$ na categoria convexa. No modo de iluminação por baixo, no que se refere ao grupo de idosos, os resultados foram os seguintes: $45,2 \%$ de julgamentos na categoria côncava; $19,1 \%$ na categoria plana; e $35,7 \%$ na categoria convexa. No que se refere ao grupo de pessoas com faixa etária menor, na categoria côncava houve $43,8 \%$ de julgamentos; $31,5 \%$ na categoria plana; e $24,7 \%$ na categoria convexa (Tabela 1).

Tabela 1 - Frequências absolutas e porcentagens da percepção categórica da máscara côncava.

\begin{tabular}{ccccc}
\hline Grupos & Iluminação & Categorias & Frequências & Porcentagens (\%) \\
\hline \multirow{3}{*}{ Idosos } & Côncava & 21 & $50,0 \%$ \\
& \multirow{2}{*}{ Por cima } & Plana & 3 & $7,1 \%$ \\
& & Convexa & 18 & $42,9 \%$ \\
\cline { 2 - 5 } & \multirow{3}{*}{ Por baixo } & Côncava & 19 & $45,2 \%$ \\
& & Plana & 8 & $19,1 \%$ \\
& \multirow{3}{*}{ Faixa etária menor cima } & Convexa & 15 & $35,7 \%$ \\
\cline { 3 - 5 } & & Côncava & 37 & $50,7 \%$ \\
& & Plana & 6 & $8,2 \%$ \\
& \multirow{2}{*}{ Por baixo } & Convexa & 30 & $41,1 \%$ \\
\cline { 3 - 5 } & & Côncava & 32 & $43,8 \%$ \\
& & Plana & 23 & $31,5 \%$ \\
& & Convexa & 18 & $24,7 \%$ \\
\hline
\end{tabular}

Fonte: Carvalho ML, et al., 2020.

No que se refere às diferenças entre os grupos nesse indicador, no modo de iluminação por cima, os resultados não revelaram diferenças significativamente relevantes entre os grupos $(p=0,97)$. Não houve, 
também, diferenças significativas entre os grupos no modo de iluminação por baixo $(p=0,26)$. Os grupos também foram analisados internamente com o objetivo de verificar se houve diferenças significativas nos julgamentos categóricos, devido a variação no modo de iluminação (por cima e por baixo). Com relação ao grupo de idosos, não houve diferenças significativas nos julgamentos categóricos em função dos modos de iluminação $(p=0,27)$. Por outro lado, houve diferença significativa nos julgamentos categóricos do grupo de pessoas de faixa etária menor, em função dos modos de iluminação $(p=0,01)$.

No último indicador da percepção de profundidade ou relevo da máscara côncava, utilizou-se o teste Kolmogorov-Smirnov para verificação da normalidade. As análises revelaram que a distribuição dos dados nos dois grupos e nos dois modos de iluminação não foi normal. Com isso, foram utilizados testes nãoparamétricos para a comparação das médias dos grupos. A média das atribuições métricas, em centímetros, da distância estimada pelos observadores da ponta do nariz até a base da máscara, com a iluminação por cima, no grupo de idosos foi 3,9 $(D P=1,8)$. Com relação ao grupo de pessoas de faixa etária menor, a média da estimativa métrica em centímetros foi $4,1(D P=2,0)$. Com a máscara côncava iluminada por baixo, a média das estimativas métricas, em centímetros, do grupo de idosos foi 3,94 $(D P=2,0)$. Semelhantemente, no grupo de pessoas de faixa etária menor, a média das estimativas métricas foi 3,94 $(D P=1,79)$ (Figura 2).

Figura 2 - Estimativas métricas, em centímetros, da profundidade ou relevo percebido da máscara côncava.

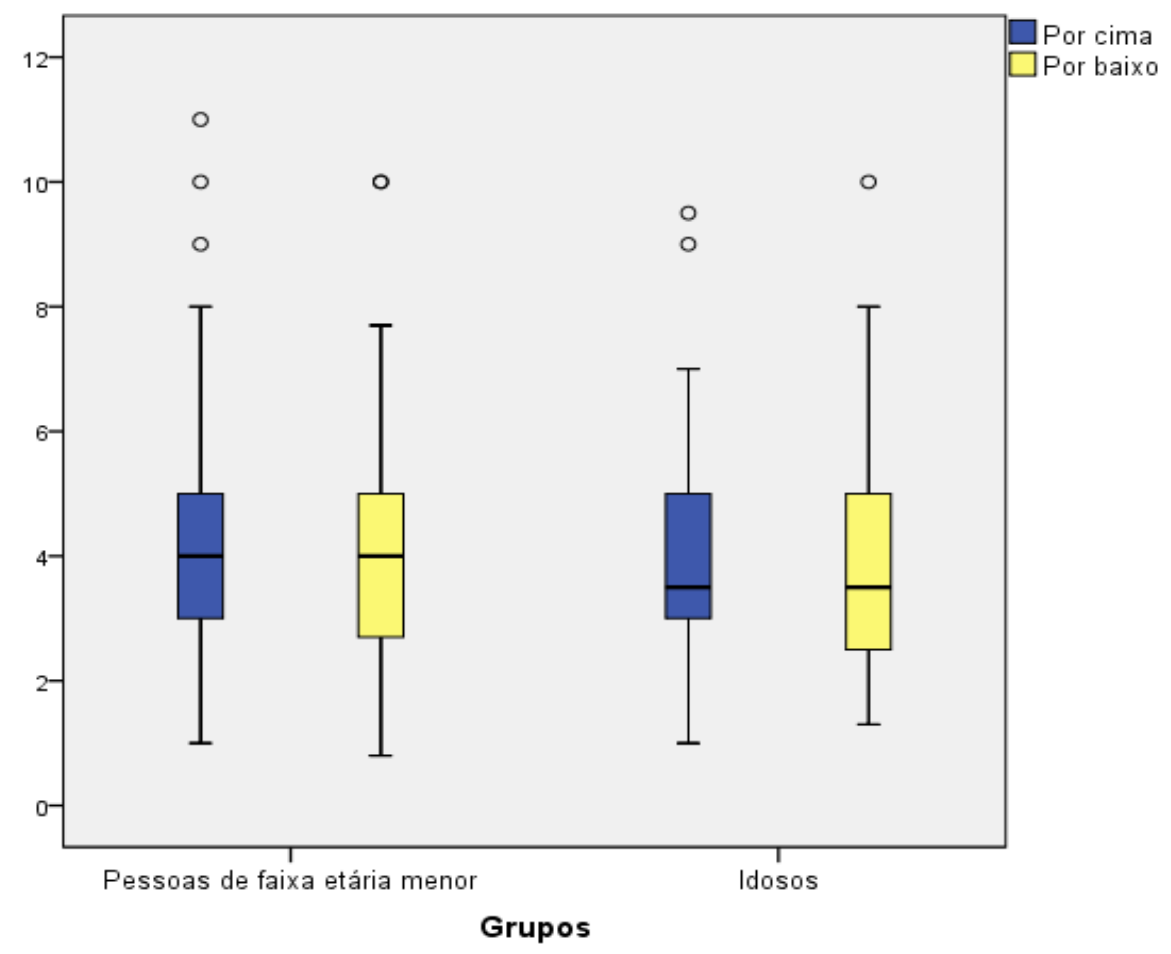

Fonte: Carvalho ML, et al., 2020.

Foram feitas análises estatísticas para verificar se houve diferenças entre os grupos nesse indicador. Os resultados não revelaram diferenças significativas entre os grupos quanto às estimativas métricas da profundidade ou relevo da máscara côncava iluminada por cima $(p=0,48)$. Não houve, também, diferenças estatisticamente relevantes entre os grupos em relação às estimativas métricas da máscara côncava iluminada por baixo $(p=0,67)$.

Os grupos foram analisados internamente para verificar possíveis diferenças significativas em função do modo de iluminação da máscara. Em relação ao grupo de idosos, os resultados do teste não revelaram diferenças significativas no que se refere às estimativas métricas da profundidade ou relevo percebido da máscara côncava em função da direção da fonte de iluminação $(p=0,72)$. Em relação ao grupo de pessoas de faixa etária menor, não foram encontradas, também, diferenças significativas quanto à estimativa métrica 
em função do modo de iluminação $(p=0,08)$, apesar do $p$-valor estar muito próximo do nível de significância adotado.

\section{DISCUSSÃO}

A percepção visual é resultado do intercâmbio de informações que ocorre entre os processos bottom-up e top-down. Na ilusão da máscara côncava, os processos top-down se sobrepõem aos processos bottom-up, fazendo com que o indivíduo inverta as pistas de profundidade vindas do ambiente (GREGORY RL, 1997a; GREGORY RL e GOMBRICH EH, 1980).

Entretanto, algumas populações específicas, como pessoas com esquizofrenia, com a síndrome da abstinência alcoólica, com uso de substâncias psicoativas e com privação do sono, apresentam dificuldades em realizar a inversão de profundidade da máscara côncava. Isso acontece porque esses fatores alteram os processos top-down impedindo sua sobreposição aos processos bottom-up (SCHNEIDER U, et al., 1996a; SCHNEIDER U, et al., 1996b; SCHNEIDER U, et al., 2002; DIMA D, et al., 2009; DIMA D, et al., 2011; BACHETTI LS, et al., 2013). Por outro lado, isso não parece ocorrer na velhice.

Alguns estudos encontraram mudanças na percepção de cores e na profundidade, reconhecimento facial e menor sensibilidade ao contraste em decorrência da velhice (MACEDO BG, et al., 2008; COSTA TL, et al., 2009; GAO L, et al., 2009). Neste estudo, não foram encontradas diferenças significativas entre pessoas idosas e pessoas de faixa etária menor no que se refere à percepção binocular da ilusão da máscara côncava. Ambas as populações apresentaram níveis de percepção semelhantes nos indicadores utilizados nesta pesquisa.

No primeiro indicador de percepção, com a máscara côncava iluminada por cima, utilizando-se a estatística descritiva, é possível perceber uma diferença sutil entre idosos e pessoas de faixa etária menor na ilusão da máscara côncava, porque os idosos apresentaram uma porcentagem menor de inversão da profundidade da máscara côncava, comparativamente ao grupo de pessoas mais jovens. Entretanto, essa diferença não foi significativa. Com a iluminação na parte inferior da máscara, os idosos apresentaram uma porcentagem maior de inversão da profundidade da máscara côncava do que quando a máscara foi iluminada na parte superior. Essa porcentagem, descritivamente, foi superior à encontrada na população de pessoas de faixa etária menor quando a máscara foi iluminada na parte inferior. No entanto, não houve diferença significativamente relevante entre os grupos.

Um dos fatores que pode ter favorecido a realização da ilusão de profundidade da máscara côncava por todos os participantes foi o fato de ter se utilizado uma máscara com tonalidades próximas a cor da pele, com a demarcação de olhos e boca. Isso foi feito com base em resultados que indicaram que quanto maior a familiaridade do objeto para o observador, mais forte será a ilusão (HILL H e JOHNSTON A, 2007). Além do mais, os resultados obtidos mostram que a ilusão da máscara côncava ocorre mesmo quando se muda a orientação da fonte de iluminação. Isso está de acordo com as pesquisas de Quaglia MAC e Fukusima SS (2009) e Alves A, et al. (2014) que demonstraram que indivíduos saudáveis realizam a ilusão da máscara côncava independentemente da direção da fonte de iluminação.

Os resultados no segundo indicador revelaram que os participantes, no momento de avaliarem a máscara, tiveram uma tendência maior em julgá-la como côncava. Isso ocorreu, semelhantemente, nos dois grupos independentemente do modo de iluminação, se por cima ou por baixo da máscara. Em outras pesquisas que utilizaram a mesma metodologia, nesse indicador, a taxa de indivíduos saudáveis que avaliaram a máscara côncava como convexa foi maior, em relação as pessoas que avaliaram a máscara como côncava (QUAGLIA MAC e FUKUSIMA SS, 2009; BACHETTI LS, et al., 2013; QUAGLIA MAC, et al., 2014; ALVES A, et al., 2014; QUAGLIA MAC E FUKUSIMA SS, 2015).

Além do mais, os resultados dos julgamentos categóricos apresentados pelo grupo de idosos não diferiram significativamente do grupo de pessoas de faixa etária menor, mesmo comparando os grupos em relação à direção da fonte de iluminação. As análises estatísticas demonstraram, ainda, que não houve diferenças internas no grupo de idosos em relação aos julgamentos categóricos conforme a mudança da fonte de 
iluminação. Porém, no grupo de pessoas de faixa etária menor, foram encontradas diferenças significativas quanto aos julgamentos categóricos, em função da direção da fonte de iluminação. Houve mais julgamentos na categoria convexa quando a máscara foi iluminada por cima.

No terceiro indicador de percepção, não foram encontradas diferenças significativas em relação aos grupos. Comparando-se as estimativas em cada grupo, em função da mudança da fonte de iluminação, também não foram encontradas diferenças significativas. De modo semelhante, as pesquisas de Bachetti LS, et al. (2013) e Quaglia MAC, et al. (2014), comparando indivíduos com a síndrome de abstinência alcóolica leve e indivíduos saudáveis, não foram encontradas diferenças entre os grupos. Por outro lado, os resultados do estudo de Alves A, et al. (2014), comparando pessoas com esquizofrenia e pessoas sem o transtorno, revelaram diferenças significativamente relevantes em relação à profundidade ou relevo da máscara côncava nesse indicador. Indivíduos com esquizofrenia sob medicação antipsicótica superior a quatro semanas julgaram a máscara côncava menos profunda.

Esta pesquisa apresenta limitação quanto à generalização dos resultados para a população idosa em geral. Pelos participantes terem sido selecionados acidentalmente e não aleatoriamente da população-alvo, torna-se difícil a generalização dos resultados.

Foi utilizada uma distância específica para a observação binocular de uma máscara côncava, de tamanho reduzido, iluminada por cima e por baixo. Considera-se importante a realização de outras pesquisas sobre a ilusão da máscara côncava, com a população idosa, utilizando outras metodologias, como apresentação de imagens estereoscópicas provindas de equipamentos eletrônicos e incluindo a teoria de detecção de sinais. Sugere-se, ainda, que os estudos avaliem a percepção monocular de idosos em relação à máscara côncava. Além disso, sugere-se mais estudos que possibilitem investigar o fenômeno de inversão da profundidade da máscara côncava em outras populações específicas, como na população infantil, trabalhadores noturnos e populações clínicas.

\section{CONCLUSÃO}

Este estudo possibilitou comparar a percepção visual de profundidade da máscara côncava entre pessoas de faixa etária menor e idosos, revelando que ambas populações são suscetíveis à ilusão da máscara côncava. Com o passar da idade, é natural que o organismo humano sofra alterações e, particularmente, o sistema visual é um dos primeiros a sofrer prejuízos, como problemas na percepção visual e doenças oftalmológicas. Todavia, neste estudo, não foram encontradas diferenças significativas entre os grupos no que se refere à ilusão da máscara côncava, sugerindo que a velhice não é um fator que prejudica a realização do fenômeno. Contudo, o fato de não ter encontrado pesquisas comparando idosos e jovens quanto à ilusão da máscara côncava, torna-se difícil compreender melhor a relação dos processos top-down e bottom-up na velhice. Por isso, estudos complementares tornam-se necessários.

\section{REFERÊNCIAS}

1. ALVES A, et al. Percepção monocular da profundidade ou relevo na ilusão da máscara côncava na esquizofrenia. Estudos de Psicologia, 2014; 19(1): 40-47.

2. BACHETTI LS, et al. Ilusão da máscara côncava na síndrome de abstinência do álcool. Arquivos Brasileiros de Psicologia, 2013; 65(3): 436-451.

3. BALDONI AO, PEREIRA LRL. O impacto do envelhecimento populacional brasileiro para o sistema de saúde sob a óptica da farmacoepidemiologia: uma revisão narrativa. Revista de Ciências Farmacêuticas Básica e Aplicada, 2011; 32(3): 313-321.

4. CONSELHO NACIONAL DE SAÚDE. Resolução no 466, de 12 de dezembro de 2012. Aprova normas regulamentadoras de pesquisa envolvendo seres humanos. Brasília: Diário Oficial da União, 2012.

5. CORROW S, et al. Six-month-old infants perceive the hollow-face illusion. Perception, 2011; 40(11):1376-83.

6. COSTA TL, et al. Envelhecimento humano e sensibilidade ao contraste fotópica para frequências angulares. Psicologia: Teoria e Pesquisa, 2009; 25(4): 589-593.

7. DARWIN C. The descent of man. New York: Prometheus, 1998 (Texto original publicado em 1871).

8. DEBERT GG. A reinvenção da velhice: socialização e processos de reprivatização do envelhecimento. São Paulo: Editora da Universidade de São Paulo, 1999. 
9. DIMA D, et al. Reduced P300 and P600 amplitude in the hollow-mask illusion in patients with schizophrenia. Psychiatry Research, 2011; 191(2): 145-51.

10. DIMA D, et al. Understanding why patients with schizophrenia do not perceive the hollow-mask illusion using dynamic causal modelling. Neuroimage, 2009; 46(4): 1180-6.

11. GAO L, et al. Aging effects on early-stage face perception: An ERP study. Psychophysiology, 2009; 1-14.

12. GREGORY RL, GOMBRICH EH. Illusion in nature and art. New York: Charles Scribner's Sons, 1980.

13. GREGORY RL. Knowledge in perception and illusion. Philosophical Transactions of the Royal Society B: Biological Sciences, 1997a; 352: 1121-1128.

14. HILL H, JOHNSTON A. The hollow-face illusion: object-specific knowledge, general assumptions or properties of stimulus? Perception, 2007; 36: 199-223.

15. LAMONT AC, et al. Face recognition and aging: Effects of target age and memory load. Memory \& Cognition, 2005; 33(6): 1017-1024.

16. MACEDO BG, et al. Impacto das alterações visuais nas quedas, desempenho funcional, controle postural e no equilíbrio dos idosos: uma revisão de literatura. Revista Brasileira Geriatria e Gerontologia, 2008; 11(3): 419-432.

17. MINISTÉRIO DA SAÚDE. (2013). Estatuto do Idoso. 3nd ed. Brasil: Brasília, DF.

18. PAPALIA EE, FELDMAN RD. Desenvolvimento humano. 12nd ed. Porto Alegre: AMGH, 2012.

19. QUAGLIA MAC, FUKUSIMA SS. Cor, iluminação e orientação do reverso de uma máscara facial não afetam a ilusão da máscara côncava. Estudos de Psicologia, 2009; 14(2): 97-105.

20. QUAGLIA MAC, et al. Ilusão da máscara côncava durante a síndrome de abstinência leve do álcool. Ciências \& Cognição, 2014; 19(3): 315-324.

21. QUAGLIA MAC, FUKUSIMA SS. Efeitos da cor, iluminação e rotação na ilusão estereoscópica da máscara côncava. Psicologia: Reflexão e Crítica, 2015; 28(2).

22. SANTOS FH, et al. Envelhecimento: um processo multifatorial. Psicologia em Estudo, Maringá, 2009; 14(1): 3-10.

23. SCHNEIDER U, et al. Reduced binocular depth inversion in schizophrenic patients. Schizophrenia Research, 2002; 53(1-2): 101-8.

24. SCHNEIDER U, et al. Impaired binocular depth inversion in patients with alcohol withdrawal. Journal of Psychiatic Research, 1996a; 30: 469-474.

25. SCHNEIDER U, et al. Visual 3D illusion: A systems - Theoretical approach to psychosis. European Archives of Psychiatry and Clinical Neuroscience, 1996b; 246: 256-260.

26. SELLTIZ C, et al. Métodos de pesquisa nas relações sociais. Delineamentos de pesquisa. V. 1. São Paulo: E.P.U., 2007. 